\title{
ORIGINAL RESEARCH \\ Minimal Hepatic Encephalopathy in Children: Evaluation with Proton MR Spectroscopy
}

\begin{abstract}
B.R. Foerster
L.S. Conklin

M. Petrou

P.B. Barker

BACKGROUND AND PURPOSE: Minimal hepatic encephalopathy (MHE) in children is difficult to evaluate because of lack of standardized neuropsychological tests for all age ranges. The purpose of this retrospective study of children with clinically suspected MHE was to investigate relationships between brain MR spectroscopy metabolites and biochemical markers of encephalopathy as well as measures of liver disease severity.
\end{abstract}

K.B. Schwarz

?

MATERIALS AND METHODS: A total of 12 children (age range, 9-19 years; 8 female) with clinically suspected MHE were studied by short TE brain MR spectroscopy on a 1.5T magnet. We estimated gray matter (GM) and white matter (WM) metabolite concentrations using "LCModel" software. Regional metabolite concentrations were examined for correlation with various parameters, including plasma ammonia, the ratio of branched-chain to aromatic amino acids (BCAA/AAA), model for end stage liver disease/pediatric end stage liver disease (MELD/PELD) and Child-Pugh scores, bilirubin, albumin, and platelet counts.

RESULTS: Myo-inositol ( $\mathrm{m} / \mathrm{ns}$ ) levels correlated with BCAA/AAA ratios $(r=0.86 ; P=.002$ for $\mathrm{GM}$ and $r=0.77 ; P=.01$ for WM). WM choline (Cho) levels and GM mlns levels showed significant negative correlation with ammonia levels $(r=-0.58 ; P=.04$ and $r=-0.65 ; P=.02$, respectively). A positive significant correlation trend was present for GM glutamine/glutamate (GIX) and ammonia levels ( $r=$ $0.66 ; P=.05)$. There was no correlation of brain MR spectroscopy parameters and severity of liver disease.

CONCLUSIONS: Brain MR spectroscopy metabolites in children with suspected MHE show significant correlations with plasma ammonia levels and BCAA/AAA. As in adults, brain MR spectroscopy in children may be helpful in establishing a diagnosis of MHE.
$\mathbf{H}^{\mathrm{e}}$ epatic encephalopathy (HE) encompasses a wide range of neuropsychiatric derangements that are associated with liver disease. In some cases, minimal or "subclinical" encephalopathy occurs, a phenomenon in which patients lack clinically obvious symptoms of encephalopathy but may have subtle symptoms and impairment on neuropsychiatric evaluations. Minimal hepatic encephalopathy (MHE) may be found in up to $70 \%$ of adult patients with cirrhosis. ${ }^{1}$ The incidence of MHE in children with liver disease is unknown. MHE is traditionally viewed as having negligible clinical significance; however, there is increasing evidence that early diagnosis and treatment is important for the preservation of brain function because not all of the effects of MHE may be reversible. ${ }^{1-3}$ This may be particularly true in the pediatric population, in which the brain is experiencing maturation and cognitive development. ${ }^{4}$

In the traditional sense, the assessment of MHE in the adult population is based on the history, physical examination, exclusion of other neurologic disorders, and the performance of neuropsychological testing. ${ }^{5-7}$ In the adult population, no clear consensus has been reached reading

Received February 9, 2009; accepted after revision March 30.

From the Russell H. Morgan Department of Radiology and Radiological Science (B.R.F., M.P., P.B.B.), and Department of Pediatrics (L.S.C., K.B.S.), Johns Hopkins University School of Medicine, Baltimore, Md.

This research was supported in part by National Institutes of Health P41 RR15241.

Please address correspondence to Bradley Foerster, MD, Division of Neuroradiology, MRI 143C, Russell H. Morgan Department of Radiology and Radiological Science, Johns Hopkins Hospital, 600 N Wolfe St, Baltimore, MD 21287; e-mail: bfoerst1@jhmi.edu

Indicates article with supplementary on-line tables.

Indicates open access to non-subscribers at www.ajnr.org

DOI 10.3174/ajnr.A1652 the neuropsychological tests required to assess MHE; furthermore, these tests can be subject to confounding factors such as age, superimposed mood disorders, and educational effects. ${ }^{8}$ These issues are magnified in the pediatric population, particularly with regard to neuropsychological testing of children at young ages.

Therefore, there has been increasing interest in the use of noninvasive imaging techniques to assist in the evaluation of MHE. In particular, MR spectroscopy has been shown to be helpful in adults for the diagnosis of both clinical and subclinical HE. MR spectroscopy of adult patients with HE has demonstrated decreases in levels of myo-inositol (mIns) and choline (Cho) and increases in the combined resonances of glutamine/glutamate (usually labeled "Glx"). ${ }^{9-11}$ Studies in adults have indicated that MR spectroscopy can detect MHE with a sensitivity approaching $90 \% .{ }^{11}$ Levels of Glx have also been shown to be significantly elevated in patients with MHE. ${ }^{11,12}$ These metabolite abnormalities have been shown to be reversible after liver transplantation. ${ }^{13,14}$

Despite these studies in adults, the value of MR spectroscopy in the assessment of MHE in children is largely unknown. ${ }^{15}$ The purpose of this study of children with clinically suspected MHE was to investigate correlations between expected MR spectroscopy findings (increased Glx, decreased mIns, and decreased Cho) and biochemical markers of encephalopathy (plasma ammonia, ratio of plasma branched-chain to aromatic amino acids (BCAA/ AAA) as well as measures of liver disease severity (ChildPugh score, model for end stage liver disease/pediatric end stage liver disease [MELD/PELD] score, serum aminotransferase levels, total and direct bilirubin, albumin, and platelet counts). 


\section{Materials and Methods}

\section{Subjects}

After obtaining institutional review board approval, we reviewed the Pediatric Liver Center data base at the Johns Hopkins Children's Center for patients with liver disease in whom MR spectroscopy was ordered in the evaluation of suspected subclinical encephalopathy. Two pediatric hepatologists ordered MR spectroscopy in patients who exhibited extreme fatigue ( 2 patients), poor attention span or school performance ( 2 patients), behavioral problems ( 1 patient), or suspected encephalopathy (7 patients).

Twelve children (age range, 9-19 years; 8 female/4 male) were identified from the data base. Mean age was $13.5 \pm 3.1$ years. Diagnoses are shown in On-line Table 1. Liver histologic studies revealed cirrhosis in 8 patients, mild to moderate periportal fibrosis in 3 patients, and portal tract/bile duct inflammation in 1 patient. Duration of liver disease at the time the MR spectroscopy was performed ranged from 2 months to 13 years. Data collected from patient charts included serum ammonia, international normalized ratio, platelet count, BCAA/AAA ratio, MELD/PELD scores (depending on patient age), Child-Pugh score, and liver chemistries. Time differences between the laboratory studies and the MR spectroscopy examination averaged $29.6 \pm 26.5$ days.

No control subjects were used for this study, given the vulnerable population protection concerns and added complexity of informed consent for pediatric subjects necessary for age-matched control subjects.

\section{MR Spectroscopy}

We performed all studies on a 1.5T Signa scanner (GE Healthcare, Milwaukee, Wis) using the standard quadrature transmit-receive head coil. In addition to routine brain MR imaging, all patients underwent short TE single-voxel spectroscopy of the medial occipital gray matter (GM) and parietal white matter (WM). The scan parameters for the point-resolved spectroscopy pulse sequence were voxel size, $8 \mathrm{~cm}^{3}$; TR, $1500 \mathrm{~ms}$; TE, $35 \mathrm{~ms}$; averages, 128 . Voxel locations are shown in Fig 1, and representative spectra from 1 subject are depicted in Fig 2. Metabolite concentrations (mIns, Cho, creatine [Cr], Glx, $\mathrm{N}$-acetylaspartate [NAA]) were estimated by "LCModel" software, ${ }^{16}$ with use of the brain water signal intensity as an internal reference. Brain MR imaging scans were reviewed to exclude other pathologic processes. All of the studies were done in patients who were conscious. The clinical setting of each patient undergoing MR spectroscopy studies as well as the overall impression of each study were also noted.

\section{Statistical Analysis}

Investigation of associations between levels of Cho, mIns, and Glx (as determined by MR spectroscopy) and plasma ammonia, BCAA/AAA levels, platelet count, and serum albumin was performed by estimation of Pearson correlation coefficients. Metabolite levels were analyzed separately for the GM and WM regions considered. A value of $P<.05$ was considered to be statistically significant.

\section{Results}

\section{MR Spectroscopy Results}

Selected clinical data are shown in On-line Table 1 with statistical averages in On-line Table 2; brain metabolite concentrations with correlations are shown in On-line Table 3 . Levels of mIns were found to have significant positive correlation with

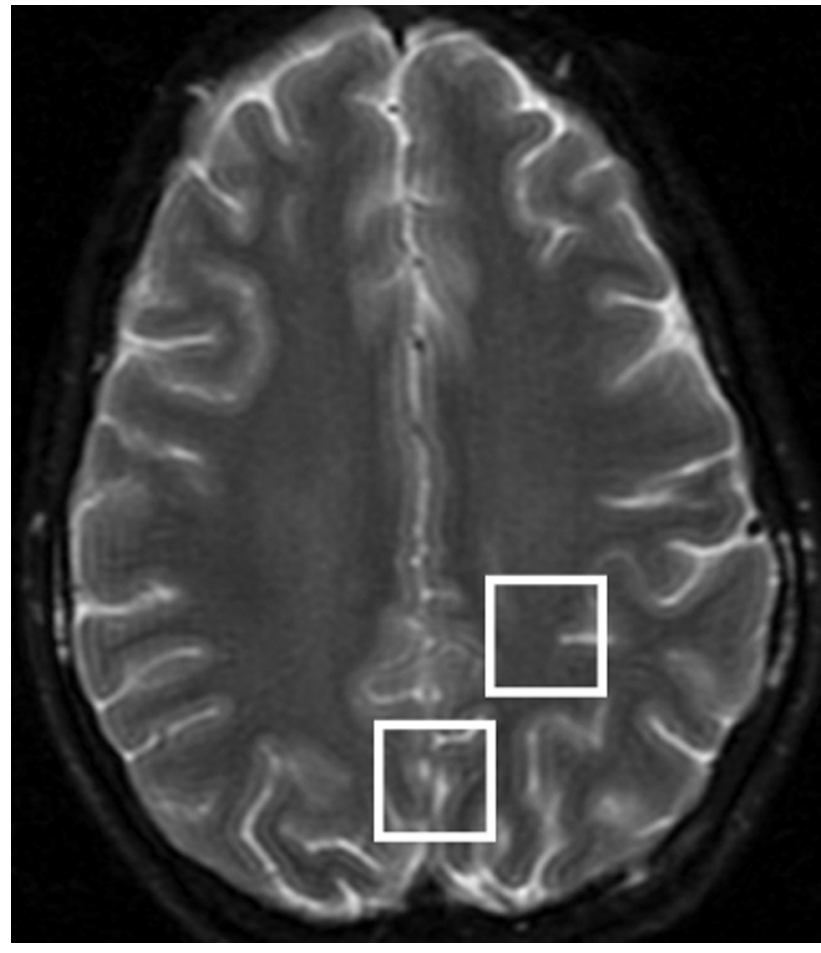

Fig $1.2 \times 2 \times 2$-cm voxel locations used for MR spectroscopy in the medial occipital GM and left parietal WM, overlaid on T2-weighted MR imaging.

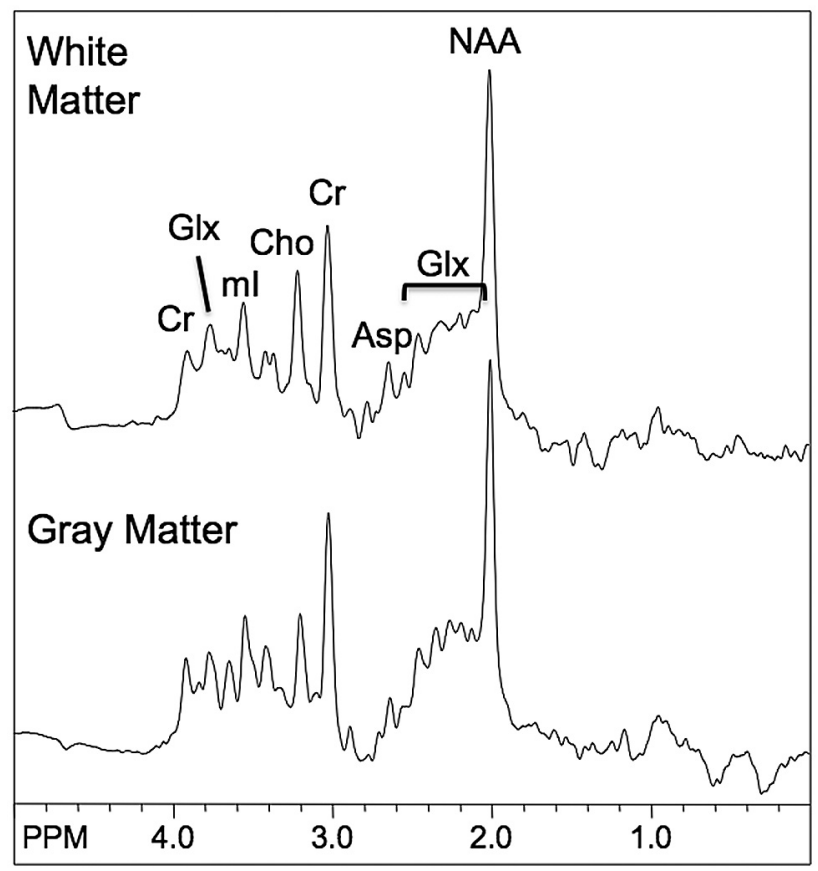

Fig 2. Examples of GM and WM spectra from one subject. Asp indicates aspartyl resonances of free aspartate and NAA.

the BCAA/AAA ratios $(r=0.86 ; P=.002$ for $\mathrm{GM}$ and $r=0.77$; $P=.01$ for WM). The WM Cho levels and GM mIns levels showed significant negative correlation with plasma ammonia levels $(r=-0.58 ; P=.04$ and $r=-0.65 ; P=.02$, respectively). Positive correlation trends were also noted for WM Glx and ammonia levels $(r=0.54 ; P=.06)$ and BCAA/AAA ratios and GM Glx $(r=0.66 ; P=.05)$. No significant correlations were present between NAA and biochemical markers or be- 
tween $\mathrm{Cr}$ and biochemical markers. No significant correlations were found between brain metabolites and serum albumin, Child-Pugh score, MELD/PELD score, or platelet count.

\section{Clinical Setting and Impression of MR Spectroscopy Studies}

Eight of the 12 cases were done in anticipation of transplant or pretransplant. Of the 12 scans interpreted by an experienced MR spectroscopist (P.B.B.), 7 patients had MR spectroscopy findings consistent with $\mathrm{HE}, 3$ showed findings consistent with mild metabolic impairment and possible HE, and 2 had results that were considered normal.

\section{Discussion}

In this series of pediatric patients with chronic liver disease and suspected MHE, the primary finding was a significant correlation between GM and WM mIns levels and plasma BCAA/ AAA ratios. The plasma Fischer ratio (molar ratio BCAA/ AAA) decreases in patients with chronic liver disease. In the study of adults by Campollo et al, ${ }^{17}$ mean values for various types of subjects were as follows: normal individuals (3.9), stable cirrhotics (2.9), unstable cirrhotics (1.7), and patients with $\mathrm{HE}$ (0.8). An imbalance in plasma levels of AAA phenylalanine, tyrosine, and tryptophan and BCAAs and their BCAA/AAA ratio has been suggested to play a causal role in HE by enhanced brain AAA uptake and subsequently disturbed neurotransmission. ${ }^{18}$ Of the variables analyzed, the strongest correlation was between the mIns and BCAA/AAA levels, suggesting that mIns levels may be affected by brain AAA uptake. The GM mIns levels showed significant negative correlation with plasma ammonia levels, which are also traditionally used as a clinical marker of HE. Previous reports have suggested that the best MR spectroscopy predictors of mild chronic HE were GM and WM mIns levels and that the observed decrease in mIns levels may be the central derangement in chronic HE. ${ }^{11}$ Although not completely understood, it has been suggested that mIns may function as an osmolite and has an important role in the volume regulation of astrocytes. ${ }^{19,20} \mathrm{It}$ is hypothesized that excess ammonia elevates intracellular levels of glutamine, causing the glial cells to swell with compensatory release of mIns by the cells. ${ }^{21,22}$

Positive correlations were also noted for WM Glx and plasma ammonia levels $(r=0.54 ; P=.06)$. Levels of Glx have been shown to have significant increases in patients with MHE. The brain lacks an effective urea cycle with cerebral ammonia removal depending on the formation of amino acids such as glutamine..$^{23,24}$ In the Glx cycle, excess ammonia is taken up by the astrocytes, which then convert glutamate to glutamine reflected in the increased in Glx peaks seen on MR spectroscopy. ${ }^{25}$ It has also been shown that liver transplantation in adults results in renormalization of abnormal mIns and Glx levels, concomitant with recovery of clinical signs of $\mathrm{HE}$ and subclinical HE, further substantiating the link between mIns and Glx levels and neuropsychological dysfunction in patients with chronic liver disease. ${ }^{13,14}$

The WM Cho levels had a significant negative correlation with ammonia levels. Previous research efforts also have reported abnormally low Cho levels in the WM, but not in the GM, in patients with MHE. ${ }^{11}$ Huda et $\mathrm{al}^{22}$ found decreased
Cho levels in the WM as well suggesting that decreased Cho levels may represent miscommunication between glial cells and cholinergic neurons. Cholines are also synthesized in the liver and transported to the brain, and the decrease in WM Cho may represent decreased liver Cho synthesis because of liver disease.

Although several studies have evaluated the relationship of MHE and HE with MR spectroscopy in the adult population, our study is the first as far as we are aware that describes MR spectroscopy findings in children. Our data suggest that MHE in children shows similar brain metabolic alterations as in adults with decreased mIns and Cho levels and increased Glx levels. These changes are proportional to biochemical markers of $\mathrm{HE}$, including plasma ammonia level and BCAA/AAA ratios. Early detection of brain metabolite alterations may be helpful in mitigating the neurocognitive declines seen in children with liver disease by consideration of liver transplantation before obvious end stage liver disease. It has been shown that in contrast to patients with adult-onset liver disease, children show deficits on global intellectual measures as well as specific neuropsychological measures, with these deficits persisting after liver transplantation. ${ }^{4}$ It has been speculated that even brief exposures to cerebrotoxic substances can accumulate during end stage liver disease. Substances such as ammonia or endogenous benzodiazepines can have a detrimental impact during early neurologic development that results in cognitive impairments, particularly in early onset of disease. ${ }^{26,27}$

When MR spectroscopy is performed, either single-voxel or multi-voxel techniques can be used at either long or short TEs. In our study, short TE was used because Glx signals decay rapidly with increasing TE. Single-voxel spectra were acquired because they can be performed in a short scan time (important for pediatric studies) and give good-quality short TE data. Two brain regions (occipital GM and parietal WM) were chosen because they are the standard locations used for MR spectroscopy in hepatic encephalopathy following the pioneering studies of Kreis et al. ${ }^{9}$ MHE is expected to have a global metabolic effect on the brain, though the tissue response may vary slightly from one brain region to another. ${ }^{28,29}$

Our study had several limitations. As mentioned before, it is difficult to institute and standardize neuropsychological testing in the pediatric population, especially given the varying cognitive and educational levels in children of different ages. In our small cohort of patients, we performed MR spectroscopy on the basis of the gestalt clinical suspicion of MHE and did not have quantifiable neuropsychological data to correlate with the MR spectroscopy results. There were time differences between the MR spectroscopy study and the laboratory values; however, the children had chronic liver failure, and we presumed that the brain imaging studies and amino acid ratios were reflective of chronic changes. Other weaknesses included the relatively wide age range of the patient cohort, though age-related changes in brain metabolites in children are very subtle after the first few years of life. ${ }^{30}$ Also, no pediatric control subjects were available for comparison, so it was not possible to establish whether metabolite levels were significantly different from those in healthy subjects. 


\section{Conclusions}

The mIns levels correlated with BCAA/AAA ratios $(r=0.86$; $P=.002$ for GM and $r=0.77 ; P=.01$ for WM). The WM Cho levels and GM mIns levels showed significant negative correlation with ammonia levels $(r=-0.58 ; P=.04$ and $r=$ $-0.65 ; P=.02$, respectively). In conclusion, proton MR spectroscopy can add an objective measure of cerebral involvement in children with symptoms suggestive of MHE and may also provide information about mechanisms of neurologic metabolic dysfunction in this subject group. Although more investigation in larger pediatric cohorts is needed, proton MR spectroscopy may one day contribute to prioritization of subjects awaiting liver transplantation.

\section{Acknowledgments}

We thank Kim Pfeiffer and Mary Kay Alford for their contributions to our study.

\section{References}

1. Stewart CA, Reivich M, Lucey MR, et al. Neuroimaging in hepatic encephalopathy. Clin Gastroenterol Hepatol 2005;3:197-207

2. Mattarozzi K, Stracciari A, Vignatelli L, et al. Minimal hepatic encephalopathy: longitudinal effects of liver transplantation. Arch Neurol 2004;61:242-47

3. Mechtcheriakov S, Graziadei IW, Mattedi M, et al. Incomplete improvement of visuo-motor deficits in patients with minimal hepatic encephalopathy after liver transplantation. Liver Transpl 2004;10:77-83

4. Stewart SM, Hiltebeitel C, Nici J, et al. Neuropsychological outcome of pediatric liver transplantation. Pediatrics 1991;87:367-76

5. Gitlin N, Lewis DC, Hinkley L. The diagnosis and prevalence of subclinical hepatic encephalopathy in apparently healthy, ambulant, non-shunted patients with cirrhosis. J Hepatol 1986;3:75-82

6. Loguercio C, Del Vecchio-Blanco C, Coltorti M. Psychometric tests and "latent" portal-systemic encephalopathy. Br J Clin Pract 1984;38:407-11

7. Watanabe A. Cerebral changes in hepatic encephalopathy. J Gastroenterol Hepatol 1998;13:752-60

8. Stewart CA, Smith GE. Minimal hepatic encephalopathy. Nat Clin Pract Gastroenterol Hepatol 2007;4:677-85

9. Kreis R, Ross BD, Farrow NA, et al. Metabolic disorders of the brain in chronic hepatic encephalopathy detected with H-1 MR spectroscopy. Radiology 1992;182:19-27

10. Ross BD, Danielsen ER, Bluml S. Proton magnetic resonance spectroscopy: the new gold standard for diagnosis of clinical and subclinical hepatic encephalopathy? Dig Dis 1996;14 Suppl 1:30-39

11. Ross BD, Jacobson S, Villamil F, et al. Subclinical hepatic encephalopathy: proton MR spectroscopic abnormalities. Radiology 1994;193:457-63
12. Binesh N, Huda A, Thomas MA, et al. Hepatic encephalopathy: a neurochemical, neuroanatomical, and neuropsychological study. J Appl Clin Med Phys 2006;7:86-96

13. Naegele T, Grodd W, Viebahn R, et al. MR imaging and (1)H spectroscopy of brain metabolites in hepatic encephalopathy: time-course of renormalization after liver transplantation. Radiology 2000;216:683-91

14. Thomas MA, Huda A, Guze B, et al. Cerebral 1H MR spectroscopy and neuropsychologic status of patients with hepatic encephalopathy. AJR Am J Roentgenol 1998;171:1123-30

15. Sijens PE, Alkefaji H, Lunsing RJ, et al. Quantitative multivoxel (1)H MR spectroscopy of the brain in children with acute liver failure. Eur Radiol 2008;18:2601-09

16. Provencher SW. Estimation of metabolite concentrations from localized in vivo proton NMR spectra. Magn Reson Med 1993;30:672-79

17. Campollo O, Sprengers D, McIntyre N. The BCAA/AAA ratio of plasma amino acids in three different groups of cirrhotics. Rev Invest Clin 1992;44:513-18

18. Dejong CH, van de Poll MC, Soeters PB, et al. Aromatic amino acid metabolism during liver failure. J Nutr 2007;137(6 Suppl 1):1579S-85S; discussion 97S-98S

19. Haussinger D, Laubenberger J, vom Dahl S, et al. Proton magnetic resonance spectroscopy studies on human brain myo-inositol in hypo-osmolarity and hepatic encephalopathy. Gastroenterology 1994;107:1475-80

20. Lee JH, Seo DW, Lee YS, et al. Proton magnetic resonance spectroscopy $(1 \mathbf{H}$ MRS) findings for the brain in patients with liver cirrhosis reflect the hepatic functional reserve. Am J Gastroenterol 1999;94:2206-13

21. Haussinger D, Kircheis G, Fischer R, et al. Hepatic encephalopathy in chronic liver disease: a clinical manifestation of astrocyte swelling and low-grade cerebral edema? J Hepatol 2000;32:1035-38

22. Huda A, Guze BH, Thomas A, et al. Clinical correlation of neuropsychological tests with $1 \mathrm{H}$ magnetic resonance spectroscopy in hepatic encephalopathy. Psychosom Med 1998;60:550-56

23. Cooper AJ, Mora SN, Cruz NF, et al. Cerebral ammonia metabolism in hyperammonemic rats. J Neurochem 1985;44:1716-23

24. Zwingmann C. Nuclear magnetic resonance studies of energy metabolism and glutamine shunt in hepatic encephalopathy and hyperammonemia. J Neurosci Res 2007;85:3429-42

25. Zwingmann C, Butterworth R. An update on the role of brain glutamine synthesis and its relation to cell-specific energy metabolism in the hyperammonemic brain: further studies using NMR spectroscopy. Neurochem Int 2005;47:19-30

26. Stewart SM, Uauy R, Kennard BD, et al. Mental development and growth in children with chronic liver disease of early and late onset. Pediatrics $1988 ; 82: 167-72$

27. Krull K, Fuchs C, Yurk H, et al. Neurocognitive outcome in pediatric liver transplant recipients. Pediatr Transplant 2003;7:111-18

28. Taylor-Robinson SD, Sargentoni J, Marcus CD, et al. Regional variations in cerebral proton spectroscopy in patients with chronic hepatic encephalopathy. Metab Brain Dis 1994;9:347-59

29. Köstler H. Proton magnetic resonance spectroscopy in portal-systemic encephalopathy. Metab Brain Dis 1998;13:291-301

30. Pouwels PJ, Brockmann K, Kruse B, et al. Regional age dependence of human brain metabolites from infancy to adulthood as detected by quantitative localized proton MRS. Pediatr Res 1999;46:474-85 